\title{
Alloy Materials for Plasmonic Refractive Index Sensing
}

\author{
Yoshiaki Nishijima, ${ }^{*}$ Yoshikazu Hashimoto, Armandas Balčytis, ${ }^{1,2}$ \\ Gediminas Seniutinas, ${ }^{1,3}$ and Saulius Juodkazis ${ }^{1}$ \\ Department of Electrical and Computer Engineering, Graduate School of Engineering, \\ Yokohama National University, 79-5 Tokiwadai, Hodogaya-ku, Yokohama, Kanagawa 240-8501, Japan \\ ${ }^{1}$ Centre for Micro-Photonics, Faculty of Engineering and Industrial Sciences, \\ Swinburne University of Technology, Hawthorn, VIC 3122, Australia \\ ${ }^{2}$ Institute of Physics, Center for Physical Sciences and Technology, \\ 231 Savanori1233u Avenue, LT-02300 Vilnius, Lithuania \\ ${ }^{3}$ Paul Scherrer Institute, Villigen CH-5232, Switzerland \\ (Received February 14, 2017; accepted May 17, 2017)
}

Keywords: plasmon sensor, alloy materials, numerical calculations

We have numerically investigated the plasmonic refractive index sensor capabilities of $\mathrm{Au}-\mathrm{Ag}-$ $\mathrm{Cu}$ alloys using experimentally determined permittivity values. Calculations were performed by simulating the typical Kretschmann configuration of surface plasmon polariton (SPR) excitation, as well as the localized surface plasmon resonance (LSPR) of nanoparticles. Sensor performance was evaluated as the response to changes in the refractive index of the surrounding environment as well as the attainable separation of peaks. By considering not only the peak shift, but also the spectral separation, SPR and LSPR sensors can be discussed on equal terms. In studies of both SPR and LSPR, specific alloy configurations were found to be superior to pure gold.

\section{Introduction}

Plasmons represent a set of optical phenomena caused by the resonance between oscillations of the electromagnetic field of incident light and collective oscillations of free electrons. When the term plasmonic materials is invoked in the visible wavelength range, we generally mean metals, in particular gold, silver, and copper.

However, for numerous applications, there is a distinct need to broaden the range of plasmonic materials and, by extension, their properties through alloying methods. Chemical synthesis is just one example of a particularly rapid way of creating various plasmonic nanoparticles. ${ }^{(1-7)}$

When estimating the optical properties of alloys, weighted averaging of the constants associated with the constituent pure metals is often attempted; however, the results are often inconsistent with observations. We first demonstrated the importance of experimental determinations of the optical constants/permittivity of metals $\left(n=n+i k, \varepsilon=\varepsilon_{1}+i \varepsilon_{2}=n^{2}-k^{2}+2 i n k\right) .{ }^{(8,9)}$

Subsequently, numerous research groups published the results of their alloy permittivity measurements. ${ }^{(10-12)}$ It was shown that optical properties were strongly dependent on the deposition method employed, and, by extension, on the resulting extent of structural order (such as the degree of crystallinity, phase separation). This was illustrated in the recent experimental investigation of permittivity in 38 variations of the $\mathrm{Au}-\mathrm{Ag}-\mathrm{Cu}$ alloy system. ${ }^{(13)}$ Furthermore, ${ }^{*}$ Corresponding author: e-mail: nishijima-yoshiaki-sp@ynu.ac.jp http://dx.doi.org/10.18494/SAM.2017.1582 
optical permittivity data can be used to predict the surface plasmon polariton (SPR)/localized surface plasmon resonance (LSPR) sensing properties of a metal.

In this study, we numerically calculated $\mathrm{Au}-\mathrm{Ag}-\mathrm{Cu}$ alloy metal SPR and LSPR properties and estimated their variations in response to changes in the refractive index of the surrounding environment. In each case, calculations were based on experimentally determined alloy permittivity values. Furthermore, since in most other publications, only the resonance peak shift per refractive index unit (RIU) change is considered, performance comparisons between angulardependence-based SPR sensors and wavelength-shift-dependence-based LSPR sensors are made difficult. ${ }^{(14-16)}$

However, the separation between two peaks can be used to draw comparisons between the two different methods. Consequently, we also discuss the sensing performance of SPR/LSPR sensors on the basis of their unified figures of merit (FOMs).

\section{Samples and Methods}

Numerical calculations of the SPR/LSPR sensor response have been performed using experimentally determined values of optical properties of the 38 different $\mathrm{Au}-\mathrm{Ag}-\mathrm{Cu}$ metal alloys published previously. ${ }^{(13)}$ Therein, optical constants were deduced by subjecting three different thickness layers for each of the alloys to measurements of the transmittance and reflectance spectra.

SPR can be induced on a flat metal film via the evanescent coupling of light. Most common methods of achieving this in SPR sensors involve the use of total internal reflection in a prism. Two types of surface plasmon excitation geometries, which use prisms, are known as Otto and Kretshmann configurations.

Since the Otto geometry requires a controlled nanometer-scale air gap between the prism and a thin metal film, the significantly simpler Kretshmann configuration is a more prevalent realization of SPR sensing. Usually, the SPR excitation angle is approximately expressed as

$$
\sin \theta \sim \frac{n_{s}}{n_{p}},
$$

where $n_{s}$ is the refractive index of the sensing layer, and $n_{p}$ is the refractive index of the prism. A rigorous expression for the asymmetric curve-fitting equation of a Kretshmann SPR sensor, derived from Fresnel equations for each of the layers (air, metal, and prism), was reported by Kurihara et al. ${ }^{(17)}$ This expression was used to simulate SPR in this work.

Obtaining stable SPR performance comparisons between the near-infrared excitation of the alloys is preferable, since both $\mathrm{Au}$ and $\mathrm{Cu}$ have absorption losses due to interband transitions in the spectral region below $600 \mathrm{~nm}$. For this reason, the wavelength of incident light was set at $800 \mathrm{~nm}$. Furthermore, the metal film thickness was set to $50 \mathrm{~nm}$. For film thicknesses below $30 \mathrm{~nm}$ (almost the same order as the skin depth of a metal), absorption losses become excessive. Conversely, above $70 \mathrm{~nm}$ thickness, reflection at the prism/metal interface becomes larger as light can no longer penetrate to the metal/air interface.

For numerical simulation of LSPR on spherical nanoparticles, absorption and scattering cross sections were obtained using the following equations. ${ }^{(18)}$ 


$$
\begin{aligned}
\sigma_{a b s} & =k \operatorname{Im}(\alpha) \\
\sigma_{s c a} & =\frac{k^{4}}{6 \pi}\left|\alpha^{2}\right| \\
\alpha & =4 \pi r^{3} \frac{\left(\varepsilon_{1}-\varepsilon_{s}\right)\left(\varepsilon_{1}+2 \varepsilon_{s}\right)+\left(\varepsilon_{2}\right)^{2}+i 3 \varepsilon_{2} \varepsilon_{s}}{\left(\varepsilon_{1}+2 \varepsilon_{s}\right)^{2}+\left(\varepsilon_{2}\right)^{2}} \\
k & =\frac{2 \pi \sqrt{\varepsilon_{s}}}{\lambda}
\end{aligned}
$$

Here, $\varepsilon_{s}$ is the permittivity of the surrounding medium $\left(=n_{s}^{2}\right.$, square of refractive index of the surrounding medium) and $r$ is the diameter of the particle. The diameter of the nanosphere was set to $30 \mathrm{~nm}$. For particles, below $10 \mathrm{~nm}$ in diameter, the refractive index of the bulk alloy metal is no longer applicable and $\varepsilon_{1}$ and $\varepsilon_{2}$ become larger than would be observed in thin film.

For both SPR and LSPR sensor calculations, the surrounding refractive index change varied from 1.0 (air) to 1.4 (typical for organic solvents such as ethylene glycol).

\section{Results and Discussion}

Figure 1(a) shows the angular dependence of the reflectance associated with SPR excitation in air ( $n$ $=1$ ) in the Kretshmann geometry. As mentioned previously, in these calculations, the excitation wavelength was $800 \mathrm{~nm}$ and metal thickness was set at $50 \mathrm{~nm}$. Almost all SPR peaks appeared in the 34 to $35^{\circ}$ angle interval, with the minimum reflectance varying between 0.1 and 0.5 . Similarly, Fig. 1(b) shows the results of the optical absorbance and scattering cross section spectra calculation for $10 \mathrm{~nm}$ metal particles. There is a strong spectral correspondence between both scattering and absorbance cross section peaks. In the $\mathrm{Au}-\mathrm{Ag}-\mathrm{Cu}$ alloy system, LSPR peaks appeared in the wavelength range between 400 and $650 \mathrm{~nm}$. Owing to the general correspondence between the two cross section spectra, the optical property of scattering was chosen for the discussion of LSPR sensing.

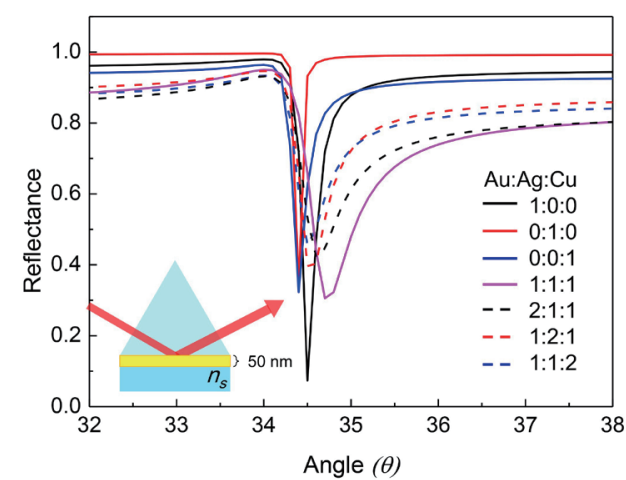

(a)
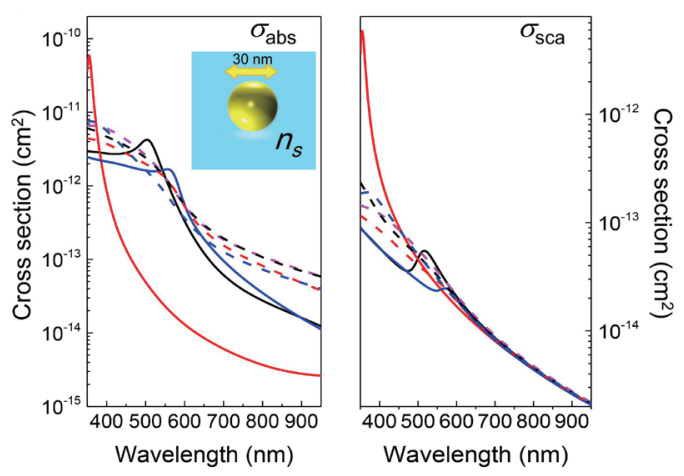

(b)

Fig. 1. (Color online) (a) Numerically calculated SPR reflection response in 50-nm-thick films of $\mathrm{Au}, \mathrm{Ag}, \mathrm{Cu}$, and several of their alloys in the Kretshmann configuration. (b) Numerically calculated spectral dependence of scattering and absorption cross sections of $10 \mathrm{~nm}$ nanoparticles of pure $\mathrm{Au}, \mathrm{Ag}, \mathrm{Cu}$, and several of their alloys. 
Figure 2 shows the variations in Au SPR and LSPR calculations caused by changes in the refractive index of the surrounding medium. Both peak angle in SPR and wavelength shift in LSPR increased linearly with increases in the surrounding refractive index. The slope values of $d \theta / d n$ and $d \lambda / d n$ are 47.8 and 77.7, respectively, for SPR and LSPR. According to these values, LSPR would appear to exhibit a higher sensitivity than an SPR sensor. However, there is a significant difference between the spectral widths of the plasmon bands of these two systems. SPR has peaks with sharp angular dependence, whereas those of LSPR are very broad. Therefore, "peak separation" is an important aspect when discussing and comparing the resolutions of the two sensing schemes. Peak separation $(R)$ is defined as

$$
R=\frac{A_{s}-A_{1}}{\frac{1}{2} \times\left(W_{s}+W_{1}\right)}
$$

where $A$ represents the position of the peak maximum either as $\theta_{\max }$ or $\lambda_{\max }$, and $W$ is the full width at half maximum of the peak. The subscripts $s$ and 1 respectively indicate the value of the refractive index in the surrounding medium and the $n=1$ condition. The red plot in Figs. 2(b)

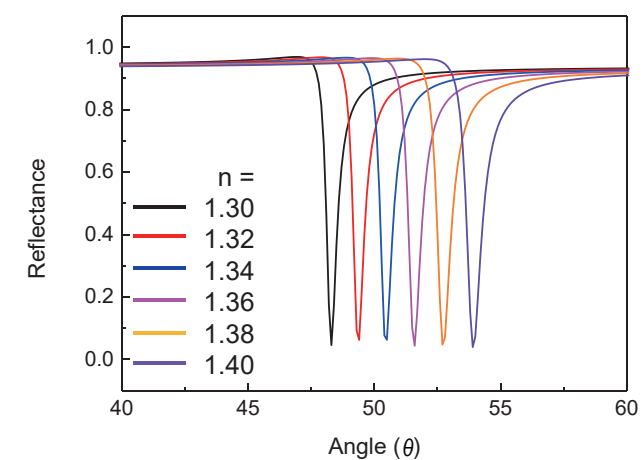

(a)

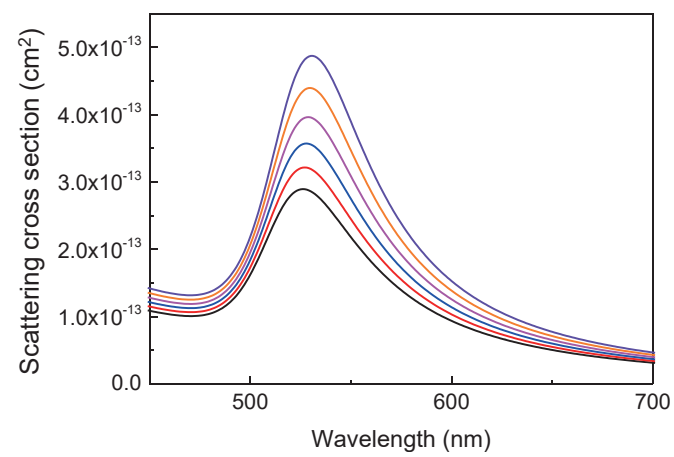

(c)

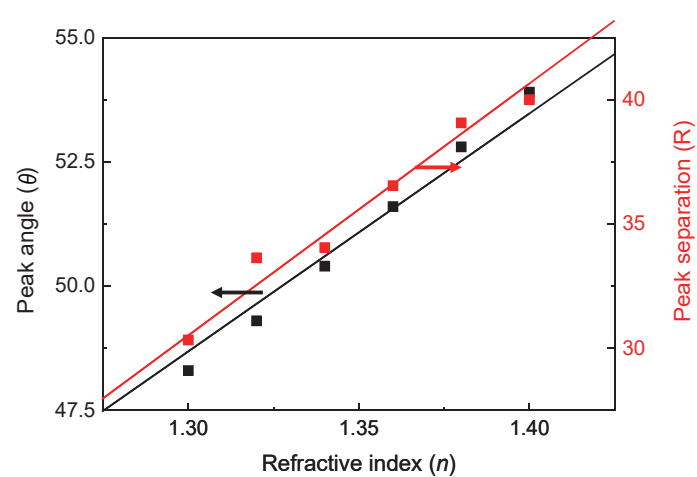

(b)

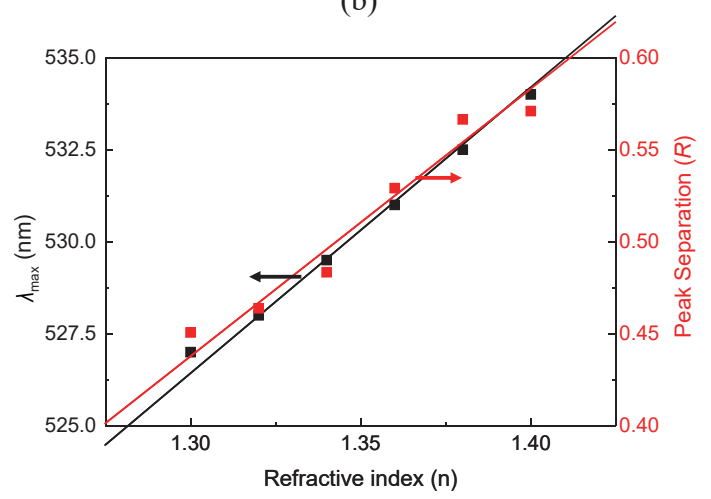

(d)

Fig. 2. (Color online) (a) Angular dependence variations of SPR excitation response as a result of changes in the refractive index of the surounding medium of a $50 \mathrm{~nm}$ Au thin film in the Kretshmann configuration. (b) Plot of the peak angular position and peak separation shifts as functions of refractive index. (c) Changes in spectral LSPR scattering cross section of a $10 \mathrm{~nm}$ Au nanoparticle as a result of variations in the refractive index of the surrounding environment. (d) LSPR peak wavelength position and peak separation shifts as functions of refractive index. 
and 2(d) shows the peak separation of the SPR and LSPR sensors. Naturally, the peak separation also linearly increases with the surrounding refractive index. However, the slopes for these plots are 101 and 1.45 for SPR and LSPR, respectively. In light of this, SPR setups have two orders of magnitude higher resolution than LSPR sensors.

The aforementioned numerical analysis of peak shift and peak separation was subsequently applied to pure $\mathrm{Au}, \mathrm{Ag}$, and $\mathrm{Cu}$ as well as to 38 different compositions of their alloys. Figure 3 shows the ternary plots of all the alloys, with panel (a) showing the peak shift and panel (b) the peak separation for the SPR configuration, whereas (c) and (d), respectively, give equivalent parameters in the LSPR case. In the case of an SPR sensor, there is a negative correlation between peak shift and peak separation. The maximum $d \theta / d n$ is observed for alloy compositions around $\mathrm{Au}_{0.6} \mathrm{Ag}_{0.1} \mathrm{Cu}_{0.3}$ and $\mathrm{Au}_{0} \mathrm{Ag}_{0.4} \mathrm{Cu}_{0.6}$, both of which are superior to pure $\mathrm{Au}, \mathrm{Ag}$, and $\mathrm{Cu}$. However, when the peak separation is considered, pure $\mathrm{Au}, \mathrm{Ag}$, and $\mathrm{Cu}$ perform far better than any of their alloys; among the alloys, $\mathrm{Au}_{0.4} \mathrm{Ag}_{0.4} \mathrm{Cu}_{0.2}$ has the largest peak separation. Conversely, for LSPR sensing, peak shift and peak separation values have a positive correlation and tend to occur simultaneously for a given alloy composition. While pure Au and Ag exhibit large FOM values-

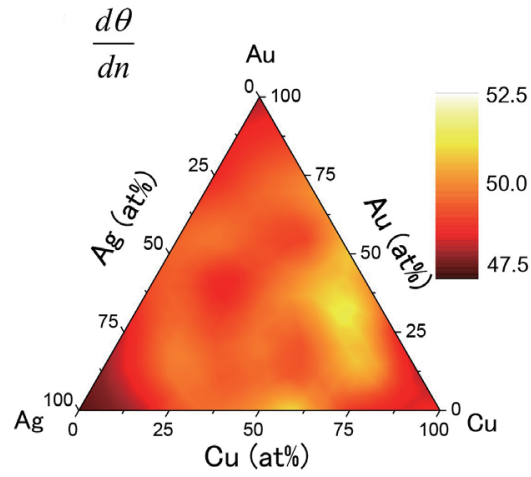

(a)

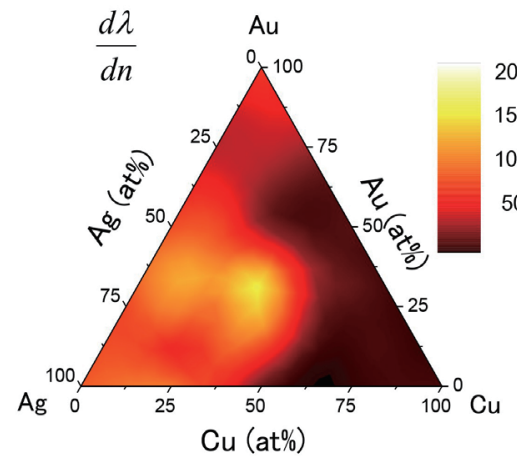

(c)

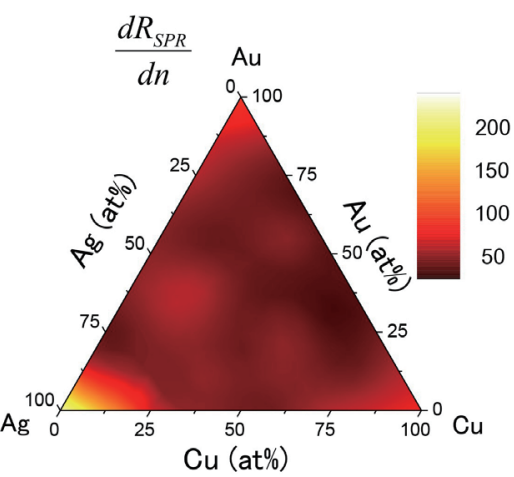

(b)

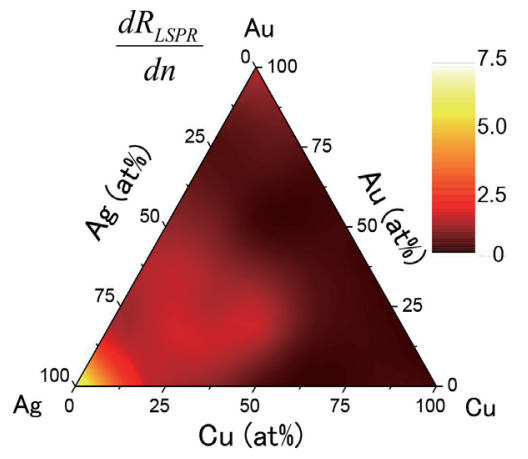

(d)

Fig. 3. (Color online) $\mathrm{Au}-\mathrm{Ag}-\mathrm{Cu}$ alloy composition map of refractive index sensing performance FOMs. (a) SPR peak angle shift in the Kretshmann configuration, (b) SPR peak separation, (c) LSPR scattering peak shift for $10 \mathrm{~nm}$ nanoparticles, and (d) LSPR peak separation. 
$\mathrm{Au}, 10-50 \%$; $\mathrm{Ag}, 35-65 \%$; and $\mathrm{Cu}, 15-40 \%$ - composit materials also have values exceeding that of pure $\mathrm{Au}$ and $\mathrm{Cu}$. In this composition range, the phase separation of $\mathrm{Ag}$ and $\mathrm{Cu}$ was suppressed by the presence of $\mathrm{Au} .^{(13)}$ A totally homogeneous alloy could be obtained. From these results, the specific compositions of alloy metals have significant potential for use in SPR or LSPR sensor applications.

Of course, the technical realization of a given sensing scheme heavily affects the attainable resolution. For instance, spectrometer resolution among other factors depends on optical setup, grating, and focal length. A small spectrometer (for example, USB2000, Ocean Photonics, Co. or Minispectrometer and PMA-12, Hamamatsu Photonics Co.) has a wavelength resolution of around 3-7 $\mathrm{nm}$. On the other hand, a long-focal-length spectrometer with a CCD detector can reach resolutions above $0.1 \mathrm{~nm}$ (1000M II; Horiba Scientific Co. or Spectra Pro SP2750; Princetone Instruments Co.).

The peak shift or peak separation are very important to know the physical or chemical meaning of the sensing principle. However, it is difficult to compare the different sensing devices. For example, SPR and LSPR are measured physical quantities that are different from each other. The FOM of a sensing device is an artificial parameter for the comparative evaluation of performance. Sometimes, for particular applications, FOMs different from the usual device sensitivity, such as the footprint, weight, price of system, maintenance cost, or power consumption, must be considered. In particular, stationary versus portable sensing devices tend to exhibit contrasting FOMs. Similarly, SPR and LSPR sensor system design may be guided by considerations beyond sensitivity.

\section{Conclusions}

In this study, we numerically demonstrate the sensing performances of SPR and LSPR schemes that use the $\mathrm{Au}-\mathrm{Ag}-\mathrm{Cu}$ alloy system based on experimentally determined values of optical permittivity. Some metal alloy compositions exhibit performance superior to their pure versions, especially $\mathrm{Au}$ and $\mathrm{Cu}$. In this way, alloys provide properties beyond that of their constituent components as well as additional new functions (catalytic, hydrogen storage, shape memory, magnetic, and others). The SPR/LSPR sensing field has matured and practical use will experience rapid progress. As the application field broadens, it will be necessary to design sensor systems with application-oriented FOMs.

\section{Acknowledgments}

YN is grateful for partial support from the Japan Society for the Promotion of Science (JSPS), Grants-in-Aid for Scientific Research, Open Partnership Joint Projects of JSPS Bilateral Joint Research Projects, the Tateishi foundation, and the Amada foundation. SJ is grateful for partial support via the Australian Research Council DP130101205 Discovery Project.

\section{References}

1 R. Rajendra, P. Bhatia, A. Justin, S. Sharma, and N. Balla: J. Phys. Chem. C 119 (2015) 5604.

2 H. Nishi, T. Torimotob, and T. Tatsuma: Phys. Chem. Chem. Phys. 17 (2015) 4042.

3 A. Bansal and S. S. Verma: Ind. J. Mater. Sci. 2014 (2014). doi.org/10.1155/2014/897125 
4 A. Bansal, J. S. Sekhon, and S. S. Verma: Plasmonics 9 (2014) 143.

5 S. Link, Z. L. Wang, and M. A. El-Sayed: J. Phys. Chem. B 103 (1999) 3529.

6 K. S. Lee and M. A. El-Sayed: J. Phys. Chem. B 110 (2006) 19220.

7 M. Valodkar, S. Modi, A. Pal, and S. Thakore: Mater. Res. Bull. 46 (2011) 384.

8 Y. Nishijima and S. Akiyama: Opt. Mater. Express 2 (2012) 1226.

9 Y. Nishijima, Y. Hashimoto, G. Seniutinas, L. Rosa, and S. Juodkazis: Appl. Phys. A 117 (2014) 641.

10 O. Pena-Rodriguez, M. Caro, A. Rivera, J. Olivares, J. M. Perlado, and A. Caro: Opt. Mater. Express 4 (2014) 403.

11 C. Gong and M. S. Leite: ACS Photon. 3 (2016) 507.

12 C. Gong, M. Rebello, S. Dias, G. C. Wessler, J. A. Taillon, L. G. Salamanca-Riba, and M. S. Leite: Adv. Opt. Mater. 5 (2017) 1600568.

13 Y. Hashimoto, G. Seniutinas, A. Balcytis, S. Juodkazis, and Y. Nishijima: Sci. Rep. 6 (2016) 25010.

14 S. Kita, S. Otsuka, S. Hachuda, T. Endo, Y. Imai, Y. Nishijima, H. Misawa, and T. Baba: IEICE Trans. Electron. E95-C (2012) 188.

15 H. Abe, M. Narimatsu, T. Watanabe, T. Furumoto, Y. Yokouchi, Y. Nishijima, S. Kita, A. Tomitaka, S. Ota, Y. Takemura, and T. Baba: Opt. Express 23 (2015) 17056.

16 Y. Nishijima, K. Ueno, S. Juodkazis, V. Mizeikis, H. Misawa, T. Tanimura, and K. Maeda: Opt. Express 15 (2007) 12979.

17 K. Kurihara, K. Nakamura, and K. Suzuki: Sens. Actuators, B 86 (2002) 49.

18 T. Okamoto and K. Kajikawa: Plasmonics-Basics and Applications- (Kodansha Co. Ltd, Tokyo, 2010) p. 72. 